\title{
Aphakia correction with retropupillary fixated iris-claw lens (Artisan) - long-term results
}

This article was published in the following Dove Press journal:

Clinical Ophthalmology

23 December 2013

Number of times this article has been viewed

\author{
Maurice Schallenberg ${ }^{1,2}$ \\ Dirk Dekowski' \\ Angela Hahn' \\ Thomas Laube ${ }^{1,3}$ \\ Klaus-Peter Steuhl' \\ Daniel Meller \\ 'Department of Ophthalmology, \\ University Hospital Essen, University \\ of Duisburg-Essen, Essen, Germany; \\ ${ }^{2}$ HELIOS Klinikum Wuppertal, \\ Wuppertal, Germany; ${ }^{3}$ Zentrum für \\ Augenheilkunde PD Dr Laube, \\ Düsseldorf, Germany
}

Purpose: To evaluate the technique, safety, and efficacy of the retropupillary implantation of iris-claw intraocular lenses in a long-term follow-up study.

Patients and methods: This retrospective study included 31 eyes of 31 patients who underwent an Artisan aphakic intraocular lens implantation between January 2006 and February 2011 at the University Hospital Essen, Essen, Germany and at the Zentrum für Augenheilkunde PD Dr Laube, Düsseldorf, Germany. Preoperative data collected included demographics, etiology of aphakia, previous surgeries, preoperative eye pathology, intraocular pressure, clinical signs of endothelial cell loss, and best corrected visual acuity. Operative data and postoperative outcomes included the best corrected visual acuity, lens position, intraocular pressure, pigment dispersion, clinical signs of endothelial cell loss, development of macular edema, and other complications.

Results: Thirty-one patients were included. The mean follow-up was 25.2 months (range: 4-48 months). The mean best corrected visual acuity postoperatively was 0.64 logarithm of the minimum angle of resolution ( $\log M A R)$ and varied from $0 \log$ MAR to $3 \log M A R$. Some patients had a low visual acuity preoperatively because of preoperative eye pathologies. In 22 patients the visual acuity improved, in two patients the visual acuity remained unchanged, and seven patients showed a decreased visual acuity. Complications were peaked pupils $(\mathrm{n}=10)$ and retinal detachment in one case. Four patients showed an iris atrophy and high intraocular pressure was observed only in one patient. Subluxation of the intraocular lens, endothelial cell loss, and macular edema were not observed.

Conclusion: The presented long-term results demonstrate that retropupillary iris-claw lens implantation is a safe and effective method for the correction of aphakia in patients without capsule support. This surgical procedure has the advantages of a posterior chamber implantation with a low intraoperative and postoperative risk profile.

Keywords: aphakia, iris-claw lens, Artisan IOL, retropupillary fixated IOL

\section{Introduction}

The implantation of an intracapsular intraocular lens (IOL) remains the best result following cataract surgery. However, after complicated cataract surgery, traumatic lens luxation, luxation of the IOL after previous cataract extraction, or severe zonulolysis, there may be insufficient remaining capsular support for either intracapsular or posterior chamber sulcus placement of the IOL. In such cases, different techniques are required for IOL fixation. These techniques include sutured scleral fixation, angle-supported anterior chamber fixation, and iris fixation, either implanted into the anterior chamber or behind the iris plane.
Correspondence: Maurice Schallenberg HELIOS Klinikum Wuppertal,

Heusnerstrasse 40, 42283 Wuppertal, Germany

Tel +492028963154

Fax +49 2028963110

Email maurice.schallenberg@helioskliniken.de 
So far there is no consensus on the indications, safety, or efficacy of these alternative techniques. The surgical procedure of an angle-supported anterior chamber IOL is safe and fast. However, the use of these lenses may cause endothelial cell loss, bullous keratopathy, and secondary glaucoma. ${ }^{1}$

The advantage of scleral-fixated IOLs is that they respect the anatomy of the eye. However, the scleral-sutured IOLs have the disadvantage of a difficult and long operation procedure with severe intraocular manipulation. The complications caused by transscleral-sutured IOLs include chronic inflammation, retinal detachment, vitreous incarceration, IOL decentration, iris-IOL contact, and pigment dispersion. ${ }^{2}$ Furthermore, the transscleral sutures can lead to conjunctival erosions, scleromalacia, and endophthalmitis. ${ }^{3}$

In 1972 Worst et al presented the first iris-claw lens (a biconvex polymethyl methacrylate [PMMA] IOL). ${ }^{4}$ This lens was fixated to the midperipheral iris, where the iris is less vascularized and less reactive. ${ }^{5}$ One of the latest versions of iris-claw lenses designed for aphakic eye is the Artisan Aphakia Model 205 (convex/concave) (Ophtec BV, Groningen, the Netherlands). Several studies exist that showed favorable visual outcome and low intraoperative and postoperative complications. ${ }^{6,7}$

However, there remains the risk of endothelial cell loss with bullous keratopathy, if the Artisan IOL is implanted into the anterior chamber. ${ }^{8}$ In this retrospective study, we evaluated the technique, safety and efficacy of the retropupillary implantation of iris-claw intraocular lenses in a long-term follow-up.

\section{Patients and methods}

This retrospective study included 31 eyes of 31 patients who underwent an Artisan aphakic IOL implantation between January 2006 and February 2011 at the University Hospital Essen, Essen, Germany and at the Zentrum für Augenheilkunde PD Dr Laube, Düsseldorf, Germany.

The preoperative data collection included demographics, etiology of aphakia, previous surgeries, and preoperative eye pathology. Preoperative and postoperative evaluations comprised objective and subjective refraction, best corrected visual acuity (BCVA), slit lamp examination, gonioscopy, Goldmann applanation tonometry, indirect fundus examination, and IOL Master (Carl Zeiss Meditec AG, Jena, Germany). Optical coherence tomography (OCT) was performed to exclude macular edema.

The Artisan lens aphakia model 205 is a monofocal onepiece convex-concave PMMA IOL with an $8.5 \mathrm{~mm}$ length, a $1.04 \mathrm{~mm}$ maximum height, and a $5.0 \mathrm{~mm}$ optical zone. The haptics attach to the iris with clips on both sides of the optic part of the lens. The haptics have fine fissures in which the iris gets enclavated. The available optical power of the Artisan aphakia model 205 ranged from +2.0 diopters (D) to $+30.0 \mathrm{D}$. The manufacturer's recommended A-constant was 115 for anterior camber implantation. We used an A-constant of 116.8 and the Sanders-Retzlaff-Kraff II formula to calculate the IOL power for retropupillary implantation.

All operations were performed by the same two surgeons under general anesthesia. In case of IOL luxation the operation was combined with pars plana vitrectomy and IOL explantation. The Artisan implantation was performed using a standardized technique that, in summary, comprised: creation of two small corneal paracenteses at 3 and 9 o'clock, preparation of a $5.5 \mathrm{~mm}$ corneo-scleral tunnel at 12 o'clock; injection of intracameral acetylcholine $1 \%$, followed by cohesive viscoelasticum into the anterior chamber; inserting the IOL through the corneo-scleral tunnel upside down, in a reversed position (convex side down); after inserting the IOL into the anterior chamber, rotating the lens such that the haptics were orientated into 3 and 9 o'clock position; holding the lens with a fixation forceps through the corneo-scleral tunnel and slipping it through the pupil area; recentering the IOL behind the iris plane; lifting the IOL against the iris plane in the way the haptics become apparent through the iris stroma; enclavation of the midperipheral iris between the claw haptics with a small spatula by applying gentle pressure; suturing the corneo-scleral wound with 10-0 nylon suture and washing the viscoelasticum out of the anterior chamber; and injection of gentamicin $20 \mathrm{mg}$ and dexamethasone $2 \mathrm{mg}$ subconjunctivally.

\section{Results}

In total, 31 eyes of 31 patients were analyzed. The age of 20 male and eleven female patients ranged from 45 to 86 years, mean 72.66 years. The follow-up ranged from 4 to 48 months, mean 25.2 months. The number of surgeries before secondary IOL implantation was $1.29 \pm 0.78$.

Sixteen eyes had a late IOL luxation after cataract surgery with intracapsular IOL implantation. All of these eyes showed a zonulolysis. Two eyes had a lens luxation after ocular trauma. Twelve eyes were aphakic either after cataract extraction because of congenital cataract or after complicated cataract surgery with extensive capsular rupture and vitrectomy. One eye developed a secondary glaucoma due to an anterior chamber lens implantation. Therefore a lens explantation was planned. The etiology of aphakia is detailed in Table 1.

Four patients had pre-existing pseudoexfoliation syndrome and five had a history of retinal detachment repair. All pre-existing ocular pathologies are outlined in Table 2. 
Table I Etiology of aphakia

\begin{tabular}{ll}
\hline Etiology of aphakia & $\begin{array}{l}\text { Number of } \\
\text { patients }\end{array}$ \\
\hline Intraocular lens luxation & 16 \\
Ocular trauma & 2 \\
Aphakia after congenital cataract & $\mathrm{I}$ \\
Complicated cataract surgery & $\mathrm{II}$ \\
Secondary glaucoma after anterior chamber lens & $\mathrm{I}$ \\
\hline
\end{tabular}

The mean preoperative BCVA was $0.85 \pm 0.42$ logarithm of the minimum angle of resolution ( $\log M A R)$. The mean postoperative BCVA was $0.64 \pm 0.62 \log$ MAR. Of the 31 eyes, 22 achieved a final BCVA better than preoperative BCVA (Figure 1). A total of two eyes achieved a final BCVA equal to that measured preoperatively, and only seven ended up with poorer BCVA. One of these patients had Korsakoff's syndrome and developed an unnoticed retinal detachment.

The goal refraction was emmetropia or a slight residual myopia. In cases with high myopia the goal refraction was adapted to the refraction of the fellow eye, generally $-3.0 \mathrm{D}$. Mean preoperative spherical equivalent (SE) refraction was $5.01 \pm 6.21$ D. This refraction decreased to a mean SE of $-0.43 \pm 1.93$ D.

During the surgery no complications were observed. All of our patients had a well-centered IOL postoperatively and during the follow-up. Postoperatively, the diagnostic pupil dilation was unaffected. Nevertheless, peaked pupils were seen in ten patients postoperatively. Four patients showed iris atrophy and one case developed a retinal detachment. An elevated intraocular pressure was observed in one case. The probability of a postoperative cystoid macular edema (CME) was examined by OCT. There was no CME observed postoperatively. In no patients was pigment dispersion seen or clinical signs of a corneal decompensation detected.

\section{Discussion}

The surgical correction of aphakic eyes without capsular support is still challenging. There has to be a careful consideration of the different treatment options, for example secondary lens implantation or correction with contact lenses or glasses. In case of a secondary lens implantation, the method

Table 2 Pre-existing ocular pathologies

\begin{tabular}{ll}
\hline Pre-existing ocular pathologies & Number of patients \\
\hline Retinal detachment & 5 \\
Pseudoexfoliation glaucoma & 4 \\
Retinal vasculitis & $\mathrm{I}$ \\
Diabetic retinopathy & $\mathrm{I}$ \\
Cone rod dystrophy & $\mathrm{I}$ \\
\hline
\end{tabular}

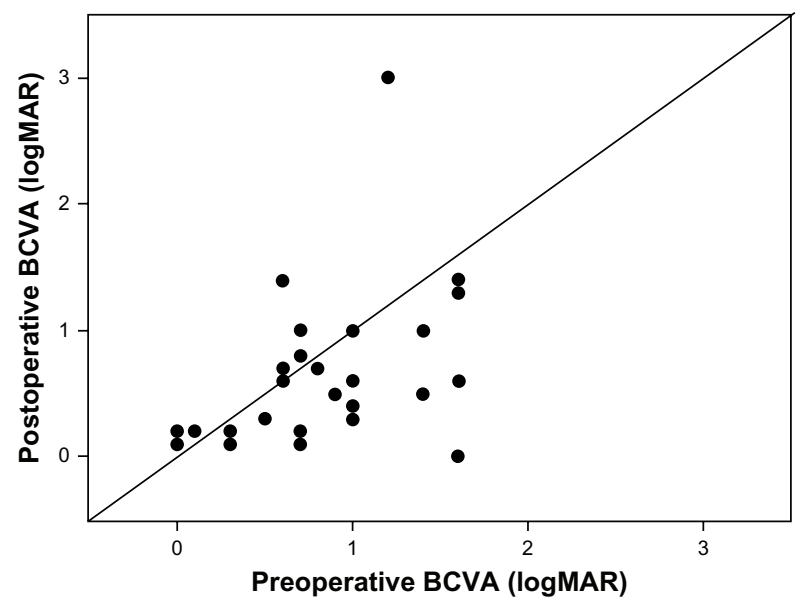

Figure I Scatter plot of preoperative BCVA versus postoperative BCVA. Abbreviations: BCVA, best corrected visual acuity; logMAR, logarithm of the minimum angle of resolution.

which offers the lowest complication rate combined with the best possible visual outcome should be chosen.

During the last two decades several studies have focused on two secondary IOL implantation techniques: angle-supported anterior chamber IOLs and scleral-sutured lenses. ${ }^{3,9}$ However, there are disadvantages to both, and there is no consensus regarding the indication for one of these techniques.

In general, the implantation of the lens into the posterior chamber behind the iris plane seems to better respect the anatomy of the eye. However, the sclera-sutured posterior chamber lens implantation has some disadvantages. In addition to early complications, such as vitreous bleeding, choroidal hemorrhage, and initial intraocular pressure fluctuation, the main risks are retinal detachment and chronic macular edema which might be caused by vitreous traction. ${ }^{1,10-12}$

In contrast to the implantation of a scleral fixated lens, the implantation of an anterior chamber lens is a time saving surgery and the operation technique is much easier. However, anterior chamber lens implantation may result in severe endothelial cell loss followed by corneal decompensation. ${ }^{13}$ Other complications include uveitis, cystoid edema, distorted pupil, and secondary glaucoma or pupillary block. ${ }^{14}$

The implantation of an iris-claw lens behind the iris plane combines the advantages of a posterior chamber lens and a short operation time as well as an easy operation technique.

In our series, we implanted the Artisan lens (model 205) retropupillary for surgical correction of aphakia. The refractive results of our patients were consistent with other groups implanting the Artisan in aphakic eyes. ${ }^{67,15}$ The mean preoperative SE was reduced from $5.01 \pm 6.21 \mathrm{D}$ to $-0.43 \pm 1.93 \mathrm{D}$ postoperatively. We used an A-constant of 116.8 and the 
SRKII formula to calculate the IOL power instead of the manufacturer's recommendation (115) which is for anterior chamber implantation. Postoperative BCVA improved in 22 of 31 patients. A total of two eyes achieved a final BCVA equal to that measured preoperatively, and only seven ended up with poorer BCVA.

If we consider only the patients without preoperative comorbidity, the BCVA improved in 13 of 17 patients (76\%). Overall, this result agrees with the results from other studies. ${ }^{7,16,17}$ Peaked pupils were seen postoperatively in ten patients but none showed a pigment dispersion. Four patients showed an iris atrophy and one case had a retinal detachment. An elevated intraocular pressure was found in one case. The probability of a postoperative CME was examined by OCT. There was no CME observed postoperatively.

Postoperative endothelial loss is seen after all cataract surgery. ${ }^{18}$ No clinical sign of a corneal decompensation was detected in any patients of our study. Koss and Kohnen reported a cumulative endothelial cell loss of $10.5 \%$ in their study where an Artisan lens was implanted into the anterior chamber in aphakic eyes. ${ }^{19}$ Another study showed a cumulative endothelial loss of $10.9 \%$ in the first 3 years after Artisan IOL implantation. ${ }^{7}$ These two studies are not directly comparable to our study, because they implanted the Artisan IOL into the anterior chamber. Two main reasons for the endothelial loss after cataract surgery and especially after anterior chamber IOL implantation were claimed. First, there is the trauma during the surgical procedure and second, a mechanical irritation from an IOL placed too close to the corneal endothelium. Therefore implantation behind the iris plane may be safer. Koss and Kohnen observed significantly smaller anterior chamber depth in smaller eyes $(<24 \mathrm{~mm})$ than in larger eyes. ${ }^{19}$ Gicquel et al directly compared patients with implantation below the iris to patients with implantation in the anterior chamber and found a significant lower endothelial cell loss in patients with posterior chamber implantation. ${ }^{20}$

The main disadvantage of the Artisan-IOL implantation is the wound size. An incision of at least $5.4 \mathrm{~mm}$ is needed for the implantation because of the single-piece PMMA material of the lens. This can lead to increased astigmatism. The mean postoperative cylinder was $-3.64 \pm 3.34 \mathrm{D}$ in our study. The new Artiflex (Ophtec BV), which allows incisions of $2.75-3.20 \mathrm{~mm}$, is able to reduce the astigmatism induced by the $5.4 \mathrm{~mm}$ incision. ${ }^{21}$ Another possible severe complication is the dislocation of the iris-claw lens into the vitreous cavity if the enclavation failed or there was inadequate tissue grasping with the haptic. We did not see this complication in our patients.
In conclusion, the presented long-term results demonstrate that retropupillary iris-claw lens implantation is a safe and effective method for the correction of aphakia in patients without capsule support. This surgical procedure has the advantages of a posterior chamber implantation with a low intraoperative and postoperative risk profile. Further studies are required to evaluate the endothelial cell loss in a long-term follow-up after retropupillary Artisan-IOL implantation.

\section{Disclosure}

The authors report no conflicts of interest in this work.

\section{References}

1. Sawada T, Kimura W, Kimura T, et al. Long-term follow-up of primary anterior chamber intraocular lens implantation. J Cataract Refract Surg. 1998;24(11):1515-1520.

2. Yilmaz A, Baser Z, Yurdakul NS, Maden A. Posterior chamber lens implantation techniques in posterior capsular rupture. Eur J Ophthalmol. 2004;14(1):7-13.

3. Kwong YY, Yuen HK, Lam RF, Lee VY, Rao SK, Lam DS. Comparison of outcomes of primary sclera-fixated versus primary anterior chamber intraocular lens implantation in complicated cataract surgeries. Ophthalmology. 2007;114(1):80-85.

4. Worst JG, Massaro RG, Ludwig HH. The introduction of an artificial lens into the eye using Binkhorst's technique. Ophthalmologica. 1972;164(5):387-391.

5. Loviosolo CF, Reinstein DZ. Phakic intraocular lenses. Surv Ophthalmol. 2005;50(6):549-587.

6. Menezo JL, Martinez MC, Cisneros AL. Iris-fixated Worst claw versus sulcus-fixated posterior chamber lenses in the absence of capsular support. J Cataract Refract Surg. 1996;22(10):1476-1484.

7. Güell JL, Velasco F, Malecaze F, Vasquez M, Gris O, Manero F. Secondary Artisan-Verysise aphakic lens implantation. J Cataract Refract Surg. 2005;3(12):2266-2271.

8. Kim M, Kim JK, Lee HK. Corneal endothelial decompensation after iris-claw phakic intraocular lens implantation. J Cataract Refract Surg. 2008;34(3):517-519.

9. Dadeya S, Kamlesh, Kumari Sodhi P. Secondary intraocular lens (IOL) implantation: anterior chamber versus sclera fixation long-term comparative evaluation. Eur J Ophthalmol. 2003;13(7):627-633.

10. Bellucci R, Pucci V, Morselli S, Bonomi L. Secondary implantation of angle-supported anterior chamber and sclera-fixated posterior chamber intraocular lenses. J Cataract Refract Surg. 1996;22(2):247-252.

11. Rattigan SM, Ellerton CR, Chitkara DK, Smerdon DL. Flexible openloop anterior chamber intraocular lens implantation after posterior capsule complications in extracapsular cataract extraction. J Cataract Refract Surg. 1996;22(2):243-246.

12. Bayramlar HS, Hepsen IF, Cekic O, Gündüz A. Comparison of results of primary and secondary implantation of flexible open-loop anterior chamber intraocular lens. Eye (Lond). 1998;12(Pt 5):826-828.

13. Hara T, Hara T. Ten-year results of anterior chamber fixation of the posterior chamber intraocular lens. Arch Ophthalmol. 2004;122(8): 1112-1116.

14. Hennig A, Johnson GJ, Evans JR, et al. Long term clinical outcome of a randomised controlled trial of anterior chamber lenses after high volume intracapsular cataract surgery. Br J Ophthalmol. 2001;85(1):11-17.

15. Mohr A, Hengerer F, Eckardt C. [Retropupillary fixation of the iris claw lens in aphakia. 1 year outcome of a new implantation techniques]. Ophthalmologe. 2002;99(7):580-583. German.

16. Lett KS, Chaudhuri PR. Visual outcomes following Artisan aphakia iris claw lens implantation. Eye (Lond). 2011;25(1):73-76. 
17. Baykara M, Ozcetin H, Yilmaz S, Timuçin OB. Posterior iris fixation of the iris-claw intraocular lens implantation through a scleral tunnel incision. Am J Ophthalmol. 2007;144(4):586-591.

18. Koch DD, Liu JF, Glasser DB, Merin LM, Haft E. A comparison of corneal endothelial changes after use of Healon or Viscoat during phacoemulsification. Am J Ophthalmol. 1993;115(2):188-201.

19. Koss MJ, Kohnen T. Intraocular architecture of secondary implanted anterior chamber iris-claw lenses in aphakic eyes evaluated with anterior segment optical coherence tomography. Br J Ophthalmol. 2009;93(10): 1301-1306.
20. Gicquel JJ, Guigou S, Bejjani RA, Briat B, Ellies P, Dighiero P. Ultrasound biomicroscopy study of the Verisyse aphakic intraocular lens combined with penetrating keratoplasty in pseudophakic bullous keratopathy. J Cataract Refract Surg. 2007;33(3):455-464.

21. Dick HB, Budo C, Malecaze F, et al. Foldable Artiflex phakic intraocular lens for the correction of myopia: two-year follow-up results of a prospective European multicenter study. Ophthalmology. 2009;116(4):671-677.

\section{Publish your work in this journal}

Clinical Ophthalmology is an international, peer-reviewed journal covering all subspecialties within ophthalmology. Key topics include: Optometry; Visual science; Pharmacology and drug therapy in eye diseases; Basic Sciences; Primary and Secondary eye care; Patien Safety and Quality of Care Improvements. This journal is indexed on

Submit your manuscript here: http://www.dovepress.com/clinical-ophthalmology-journal

\section{Dovepress}

PubMed Central and CAS, and is the official journal of The Society of Clinical Ophthalmology (SCO). The manuscript management system is completely online and includes a very quick and fair peer-review system, which is all easy to use. Visit http://www.dovepress.com/ testimonials.php to read real quotes from published authors. 